University of Nebraska - Lincoln

DigitalCommons@University of Nebraska - Lincoln

June 2007

\title{
Temperature-dependent orbital-moment anisotropy in dilute magnetic oxides
}

\author{
Jun Zhang \\ University of Nebraska - Lincoln, jzhang5@unl.edu \\ Ralph Skomski \\ University of Nebraska-Lincoln, rskomski2@unl.edu \\ Yongfeng Lu \\ University of Nebraska - Lincoln, ylu2@unl.edu \\ David J. Sellmyer \\ University of Nebraska-Lincoln, dsellmyer@unl.edu
}

Follow this and additional works at: https://digitalcommons.unl.edu/physicssellmyer

Part of the Physics Commons

Zhang, Jun; Skomski, Ralph; Lu, Yongfeng; and Sellmyer, David J., "Temperature-dependent orbitalmoment anisotropy in dilute magnetic oxides" (2007). David Sellmyer Publications. 200.

https://digitalcommons.unl.edu/physicssellmyer/200

This Article is brought to you for free and open access by the Research Papers in Physics and Astronomy at DigitalCommons@University of Nebraska - Lincoln. It has been accepted for inclusion in David Sellmyer Publications by an authorized administrator of DigitalCommons@University of Nebraska - Lincoln. 


\title{
Temperature-dependent orbital-moment anisotropy in dilute magnetic oxides
}

\author{
Jun Zhang, ${ }^{1,2}$ Ralph Skomski, ${ }^{1,2}$ Y. F. Lu, ${ }^{3,2}$ and D. J. Sellmyer ${ }^{1,2}$ \\ ${ }^{1}$ Department of Physics and Astronomy, University of Nebraska, Lincoln, Nebraska 68588, USA \\ ${ }^{2}$ Nebraska Center for Materials and Nanoscience, University of Nebraska, Lincoln, Nebraska 68588, USA \\ ${ }^{3}$ Department of Electrical Engineering, University of Nebraska, Lincoln, Nebraska 68588, USA
}

(Received 1 March 2007; published 13 June 2007)

\begin{abstract}
A striking magnetization anisotropy in thin films of vanadium-doped stannic oxide is reported and investigated. The single-crystalline $\mathrm{Sn}_{1-x} \mathrm{~V}_{x} \mathrm{O}_{2}(0 \leqslant x \leqslant 0.1)$ thin films, grown on $\mathrm{Al}_{2} \mathrm{O}_{3}$ substrate, are (101) oriented and exhibit a temperature-dependent in-plane anisotropy of the saturation magnetization. The in-plane mag-

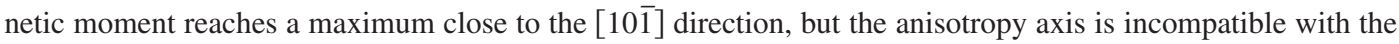
crystalline structure of the $\mathrm{SnO}_{2}$ thin films. However, it is consistent with $\mathrm{V}$ atoms occupying uniaxially distorted octahedral interstices, thus breaking the symmetry of the film. The moment anisotropy decreases gradually with increasing temperature and persists to temperatures above room temperature. It is modeled as a spin-orbit effect involving the hopping of orbital-current loops, as contrasted to isotropic Heisenberg exchange, and the temperature dependence of the magnetism is explained on the basis of a thermal admixture of nearly degenerate crystal-field states. The phenomena may be relevant to a wide range of dilute magnetic oxides.
\end{abstract}

DOI: 10.1103/PhysRevB.75.214417

PACS number(s): 75.50.Pp, 73.90.+f, 75.30.Gw

\section{INTRODUCTION}

High-temperature dilute magnetic oxide semiconductors, such as $\mathrm{Mn}$ - and Co-doped $\mathrm{ZnO}, \mathrm{TiO}_{2}$, and $\mathrm{SnO}_{2},{ }^{1-11}$ have attracted much attention in spin electronics, because they combine optical transparency and semiconductivity with ferromagnetism above room temperature. However, the ferromagnetism of these transition-metal-doped oxides is rather poorly understood. Difficult issues include the high Curie temperature ${ }^{6-9}$ the origin of large and anisotropic saturation magnetization in some systems, ${ }^{7,9}$ and the specific role of imperfections such as oxygen vacancies, substitutional disorder, and interstitial occupancies. ${ }^{4-7,11}$ These questions have far-reaching ramifications. For example, the anisotropic magnetic moment observed in Co-doped $\mathrm{ZnO}$ (Ref. 7) is incompatible with Heisenberg exchange, $J_{i j} \mathbf{s}_{i} \cdot \mathbf{s}_{j}$, and indicates the involvement of spin-orbit coupling. ${ }^{7,12,13}$ An additional challenge is that the oxides' finite-temperature magnetism may not be universal, but depend on the oxygen stoichiometry and on the electronic structure and site occupancy of the individual dopants.

The anisotropy of the moment or magnetization is usually very small and different from the widespread magnetocrystalline anisotropy, where the magnetic energy depends on the magnetization direction. For example, hexagonal Co exhibits a relatively strong magnetocrystalline anisotropy of about $0.5 \mathrm{MJ} / \mathrm{m}^{3}$, but its moment anisotropy is only about $0.5 \%$. The moment anisotropy cannot be explained by the ordinary Heisenberg exchange, which is isotropic even in anisotropic crystalline environments. Both the ordinary magnetocrystalline anisotropy and the moment anisotropy are caused by spin-orbit coupling $\lambda \mathbf{I} \cdot \mathbf{s}$, where $\mathbf{I}$ and $\mathbf{S}$ are the orbital and spin moments, respectively. ${ }^{12,13}$ In an extreme limit, the resulting anisotropic Heisenberg coupling corresponds to the classical exchange $J \cos \theta_{1} \cos \theta_{2}$, as opposed to the familiar form $J \cos \left(\theta_{1}-\theta_{2}\right)$.

In this paper, we report a moment anisotropy in a dilute magnetic oxide, V-doped $\mathrm{SnO}_{2}$. We measure the anisotropy and its temperature dependence, and model the observed behavior in terms of crystal-field interactions and spin-orbit coupling.

\section{SAMPLE PREPARATION AND CHARACTERIZATION}

Our $\mathrm{Sn}_{1-x} \mathrm{~V}_{x} \mathrm{O}_{2}$ films $(x \leqslant 0.10)$ were grown by pulsedlaser deposition (PLD) on r-cut $\mathrm{Al}_{2} \mathrm{O}_{3}$ (11 102$)$ and have thicknesses of about $50 \mathrm{~nm}$. Atomic force microscopy analysis with a vertical resolution of about $0.5 \AA$ shows that the substrate is free of steps. Step-induced surface anisotropies, which are important in other systems, ${ }^{14,15}$ can therefore be excluded. The V-doped $\mathrm{SnO}_{2}$ ceramic targets were prepared by standard solid-state reaction. The base pressure of the PLD chamber was $3 \times 10^{-7}$ Torr; during film growth, the oxygen pressure was $5 \times 10^{-4}$ Torr, and the substrate was held at $600{ }^{\circ} \mathrm{C}$. The $\mathrm{V}$ concentrations were determined by energy dispersive $\mathrm{x}$-ray spectroscopy, and the structures of the films were characterized by $\mathrm{x}$-ray diffraction (XRD). The magnetic measurements were performed with a superconducting quantum interference device (SQUID) magnetometer.

Figure 1(a) shows the XRD $\theta-2 \theta$ scan pattern of the $\mathrm{Sn}_{0.9} \mathrm{~V}_{0.1} \mathrm{O}_{2}$ thin film. The film has the rutile structure with a strong (101) texture, and no secondary phase is observed. XRD pole figures (not shown here) indicate that the film is single crystalline and is epitaxially grown with its [010] axis along the $[1 \overline{2} 10]$ direction of the $\mathrm{Al}_{2} \mathrm{O}_{3}$ substrate, consistent with the previous report in Ref. 16. The dependence of the (101) plane spacing, $d_{101}$, on the $\mathrm{V}$ concentration is shown in Fig. 1(b). The spacing decreases almost linearly with increasing $\mathrm{V}$ concentration, suggesting that the vanadium goes into the lattice rather than forming clusters outside the crystal. However, the symmetry of magnetic moment (see below) speaks against a random substitutional solid solution of $\mathrm{V}$ for Sn. 

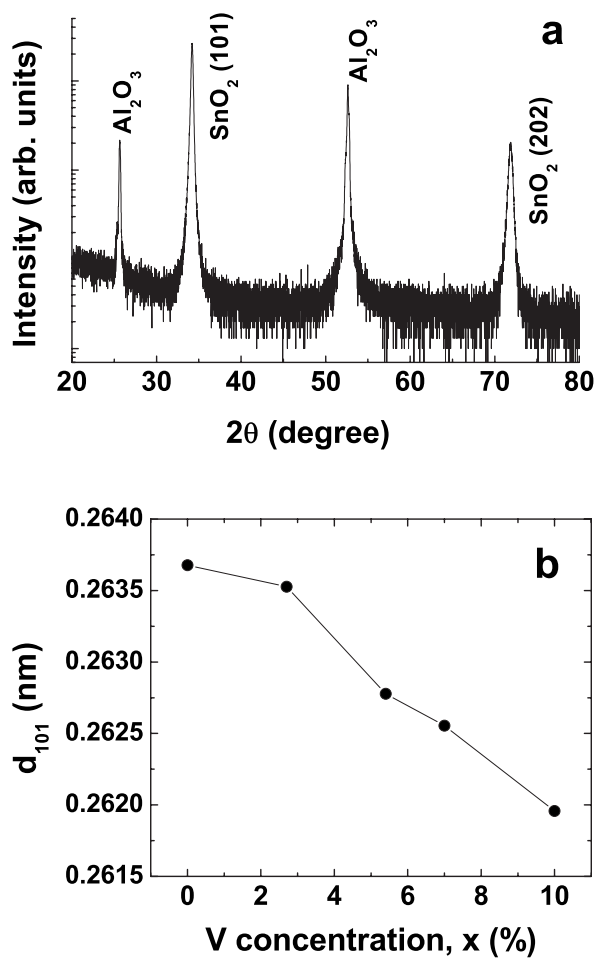

FIG. 1. $\mathrm{Sn}_{0.9} \mathrm{~V}_{0.1} \mathrm{O}_{2}$ thin-film structure: (a) $\mathrm{Cu} K \alpha$ XRD pattern and (b) $\mathrm{V}$ concentration dependence of the (101) plane spacing, $d_{101}$.

\section{MAGNETIC MEASUREMENTS}

The $\mathrm{Sn}_{1-x} \mathrm{~V}_{x} \mathrm{O}_{2}$ films exhibit a net magnetization at and above room temperature, indicative of a strong ferromagnetic coupling. Figure 2 shows $\mathrm{Sn}_{0.9} \mathrm{~V}_{0.1} \mathrm{O}_{2}$ roomtemperature hysteresis loops for magnetic fields in the film plane, parallel and perpendicular to the crystallographic [010] direction. In both directions, remanence ratio and coercivity are about $30 \%$ and 200 Oe, respectively. The coercivity indicates the presence of magnetocrystalline anisotropy, but due to the unknown coercivity mechanism, it is difficult to draw quantitative conclusions from the hysteresis loop.

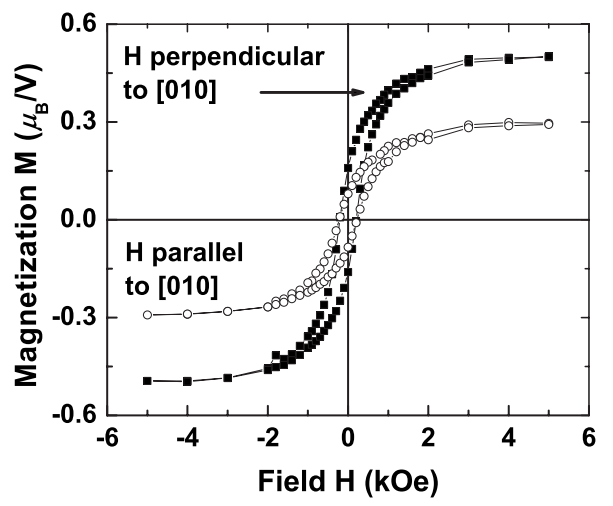

FIG. 2. Room-temperature in-plane hysteresis loops for the $\mathrm{Sn}_{0.9} \mathrm{~V}_{0.1} \mathrm{O}_{2}$ thin film with the magnetic field parallel and perpendicular to the [010] direction of the film.

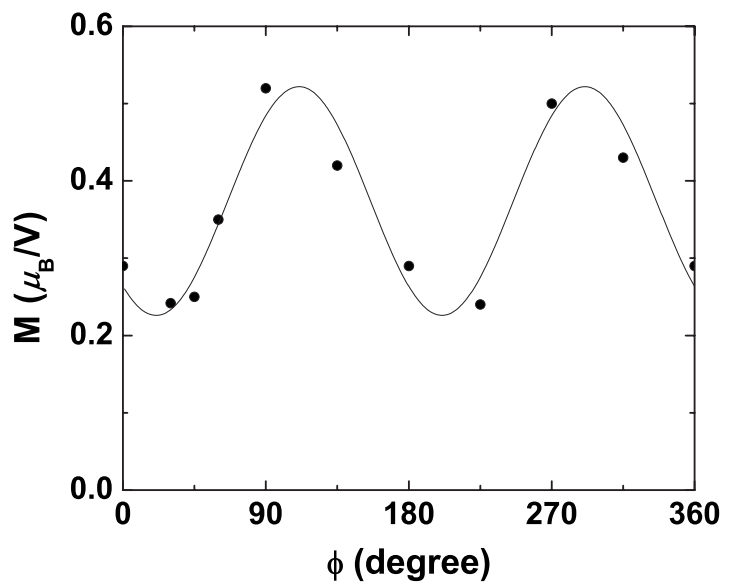

FIG. 3. Saturation magnetization in $\mu_{B} / \mathrm{V}$ atom as a function of angle $\phi$, the angle between the [010] direction and the direction of applied magnetic field. The dots are experimental data and the line is a fitting.

The saturation magnetization exhibits a pronounced inplane anisotropy of the magnetic moment. Figure 3 shows the room-temperature saturation magnetization as a function of the angle $\phi$ between the [010] direction, as determined from the pole-figure data, and the magnetization. The maximum and minimum are close to the [101] and [010] directions, respectively. The solid curve in Fig. 3 is the secondorder moment anisotropy $M(\phi)=M_{o}-\Delta M \cos \left(2 \phi-2 \phi_{o}\right)$, where $M_{o}=0.374 \mu_{B} / \mathrm{V}$ atom and $\Delta M=0.148 \mu_{B} / \mathrm{V}$ atom were obtained by fitting the experimental data. The temperature dependence of the moment anisotropy is shown in Fig. 4.

A striking feature of the moment anisotropy of Fig. 3 is the shift of the curve by an angle $\phi_{o}=20.9^{\circ}$. This shift is incompatible with both the sample geometry and the crystal structure of rutile $\mathrm{SnO}_{2}$. The sample is square and should not yield uniaxial anisotropy in any direction, whereas the crystal structure of the film does not support magnetization extrema at angles other than 0 and $\pi / 2$. However, arbitrary angles may be caused by structural features that break the symmetry of the film.

\section{ORIGIN OF MOMENT ANISOTROPY}

A possible symmetry-breaking mechanism is the formation of bonds of the type $\mathrm{V}-\mathrm{O}-\mathrm{V}$ by substitutional impurities. Such bonds are not unexpected in magnetic semiconductors, because they may be energetically favorable. ${ }^{17}$ However, in the case of $\mathrm{V}$ in $\mathrm{SnO}_{2}$, there is a tendency towards interstitial occupancy of the V. ${ }^{18,19}$ Figure 5 shows the corresponding interstitial coordination octahedron. The symmetry axis of the octahedron, shown by the dashed line, is neither parallel to the $a, b$, and $c$ axes of the rutile structure nor to the $[10 \overline{1}]$ and [010] symmetry axes in the film plane.

As evidenced by bulk paramagnetic resonance, ${ }^{18}$ the strongly distorted interstitial octahedron gives rise to a crystal field that breaks the cubic $e_{g}-t_{2 g}$ symmetry of the undoped $\mathrm{SnO}_{2}$. In the coordinate frame of Ref. 18, which differs from 


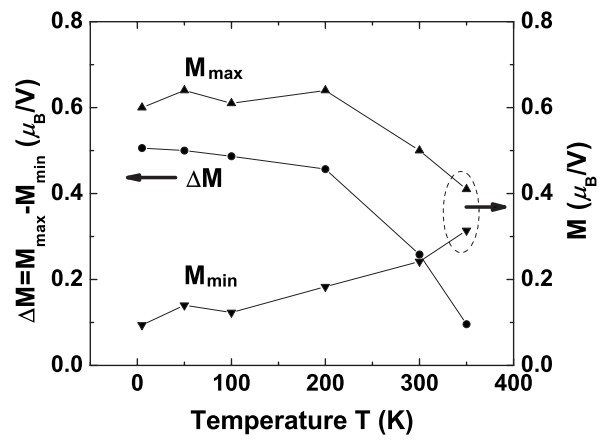

FIG. 4. Temperature dependence of the maxima and minima in-plane magnetic moments, as well as their difference, for the $\mathrm{Sn}_{0.9} \mathrm{~V}_{0.1} \mathrm{O}_{2}$ thin film.

the usual frame by a rotation of $45^{\circ}$ in the $x-y$ plane, the $t_{2 g}$ ground-state triplet is split into a low-lying $x^{2}-y^{2}$ singlet and a doublet containing the $x z$ and $y z$ levels. The large splitting, of order $3 \mathrm{eV}$, means that the energy of the doublet is nearly degenerate with the $x y$ level. This pushes the $x z$ and $y z$ levels close to the Sn conduction band, where they can hybridize with the relatively diffuse ${ }^{19} \mathrm{Sn} 5 s$ and $5 p$ orbitals. Spin-orbit coupling mixes the levels, $\left|\psi_{ \pm}\right\rangle \sim|x z\rangle \pm i|y z\rangle$, and yields an orbital moment along the symmetry axis of the octahedron. To realize the moment, the doublet must be occupied by one electron. For $\mathrm{V}^{4+}$, this is not satisfied, because the only occupied level would then be the low-lying $x^{2}-y^{2}$ state. Such occupancy carries a paramagnetic signature, is unlikely to support ferromagnetic coupling, and shows zero moment anisotropy.

Note, however, that the level occupancy is only approximately known and strongly affected by oxygen stoichiometry. The maximum orbital moment due to this mechanism, $1 \mu_{B} / \mathrm{V}$ atom, is consistent with our experimental data. Changing the stoichiometry by annealing in oxygen or vacuum enhances or reduces the anisotropy, respectively, and we have also seen some anisotropy reduction on roomtemperature aging on a time scale of several months. In the light of the notorious defect-structure and stoichiometry dependences of the properties of dilute magnetic semiconductors, ${ }^{11}$ these findings are not surprising but remain to be explained.

The orbital-moment directions obtained by projecting the dashed line in Fig. 5 onto the film plane are consistent with the observed magnetization. Since there are several octahedral interstices per unit cell, the actual moment angle depends on the unknown occupancy of these sites. However, a random interstitial occupancy with zero net effect is unlikely, because the simultaneous occupancy of interstitial sites of the type $(1 / 2,0,0)$ and $(1 / 2,0,1 / 2)$ is sterically unfavorable.

Our explanation assumes a spin-orbit-induced hybridization of the $x z$ and $y z$ levels, but, as mentioned, the bulk $x y$, $x z$, and $y z$ levels are close together and easily remixed, for example, by film strain. This may change the direction of the orbital moment by $90^{\circ}$ but leaves the basic mechanism unchanged, including the key role of the crystal field acting on the impurity atoms. The energies of the three levels are dif-

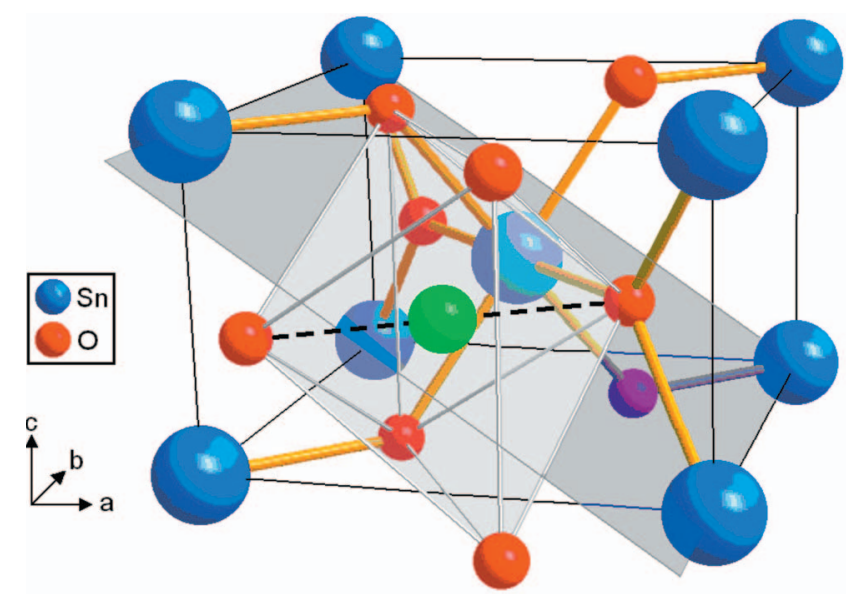

FIG. 5. (Color) Interstitial occupancy of $\mathrm{V}$ (green) in $\mathrm{SnO}_{2}$. The figure shows the location of interstitial octahedron (gray) relative to the crystal axes and to the film plane.

ficult to determine, so further analysis of this point is not possible at this stage.

\section{TEMPERATURE DEPENDENCE}

It is well known that the orbital moment reflects the competition between spin-orbit coupling and crystal-field interactions. To describe the temperature dependence of the moment anisotropy, we model the interstitial $\mathrm{V}$ atom by the single-ion Hamiltonian

$$
\mathrm{H}=\left(\begin{array}{cc}
E_{x z} & i \lambda\langle s\rangle \cos \theta \\
-i \lambda\langle s\rangle \cos \theta & E_{y z}
\end{array}\right) .
$$

Here, $\lambda$ is the spin-orbit coupling parameter, $\langle s\rangle$ is the average spin of the electron occupying the $x z$ and $y z$ states, and $\theta$ is the angle between spin and orbital moment. In elemental iron-series ferromagnets, the spin-orbit coupling $\lambda$ $\sim 0.05 \mathrm{eV}$ is a small perturbation to the one-electron level splitting of order $1 \mathrm{eV}$, and one obtains orbital-moment corrections of order $\lambda /\left(E_{x z}-E_{y z}\right)$. In the present model, the (nearly) degenerate character of the $x y$ and $x z$ states amounts to a disproportionally large spin-orbit effect, described by the levels

$$
E_{ \pm}= \pm \sqrt{\frac{1}{4}\left(E_{x z}-E_{y z}\right)^{2}+\lambda^{2} \cos ^{2} \theta\langle s\rangle^{2}} .
$$

Here, the sign \pm refers to the two eigenstates $\left|\psi_{ \pm}\right\rangle$of Eq. (1), which correspond to orbital moments of opposite directions.

At zero temperature, only the lowest-lying state $\left|\psi_{+}\right\rangle$or $\left|\psi_{-}\right\rangle$is realized, depending on the spin direction (angle $\theta$ ). The temperature dependence of the moment anisotropy has two contributions. First, with increasing temperature, both levels become occupied, in accordance with the Boltzmann factors $p_{ \pm} \sim \exp \left( \pm \Delta E / k_{B} T\right)$, and the orbital moment decreases as $f\left(\Delta E / k_{B} T\right) \sim\left(p_{+}-p_{-}\right) /\left(p_{+}+p_{-}\right)$. Second, the average spin $\langle s\rangle$ exhibits a temperature dependence corresponding to an isotropic Heisenberg exchange. A comprehensive 


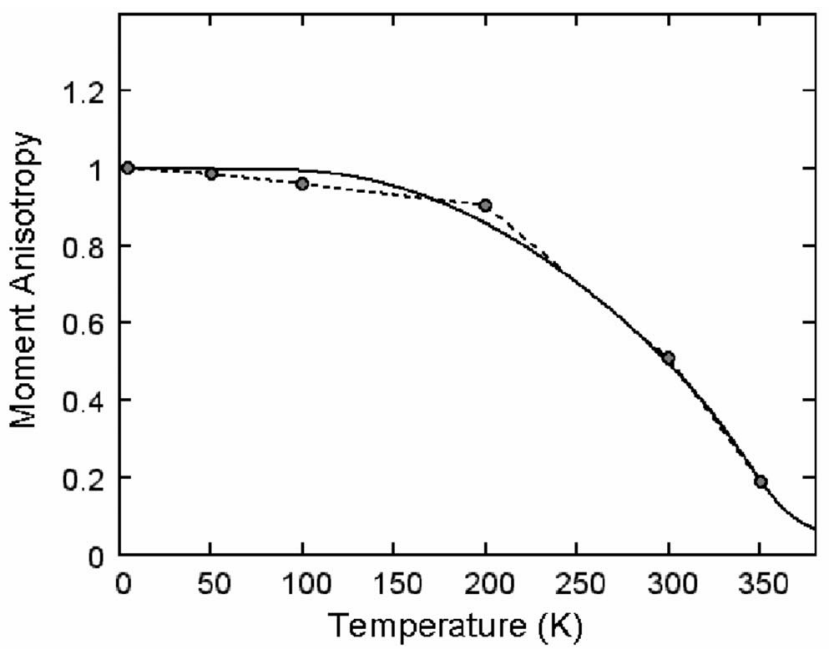

FIG. 6. Temperature dependence of the normalized moment anisotropy $\Delta M(T) / \Delta M(0)$. The experimental data points are taken from Fig. 4 and the solid curve is the model prediction for $|\Delta E|$ $=57 \mathrm{meV}$ and $J^{*}=30 \mathrm{meV}$.

description would include the temperature dependence up to the Curie temperature $T_{C}$, which could not be measured due to reduced SQUID sensitivity on installing a furnace. Here, we determine $\langle s\rangle$ from a simple two-sublattice model ${ }^{13,20}$ characterized by a coupling energy $J^{*}<k_{B} T_{C}$. The two contributions are qualitatively similar and therefore difficult to separate. Figure 6 compares the experimental temperature dependence of the moment anisotropy with $f\left(\Delta E / k_{B} T\right)$.

\section{DISCUSSION AND CONCLUSIONS}

While not attempting to provide a comprehensive explanation, we now discuss how the anisotropic orbital moment affects the interatomic coupling. As in the model of Sec. V, we consider two orbitals per atom, leading to a $4 \times 4$ matrix for a pair of interacting atoms. For simplicity, we restrict ourselves to tightly bound xy and $x^{2}-y^{2}$ orbitals and assume that the atoms are homonuclear and located in the $x-y$ plane. Note that the model is easily generalized to heteronuclear orbitals involving $\mathrm{V}, \mathrm{Sn}$, and $\mathrm{O}$ atoms, and to hybridization involving $s, p$, and $d$ electrons.

As in the isotropic Heisenberg model, ${ }^{21}$ the net exchange depends on the interatomic hopping, but the spin-orbit coupling changes the nature of the hopping. In the simplest case, the model Hamiltonian is

$$
\mathrm{H}=\left(\begin{array}{cccc}
0 & 2 i \lambda \cos \theta_{1} & t & 0 \\
-2 i \lambda \cos \theta_{1} & 0 & 0 & t \\
t & 0 & 0 & 2 i \lambda \cos \theta_{2} \\
0 & t & -2 i \lambda \cos \theta_{2} & 0
\end{array}\right) \text {, }
$$

where $t$ is the Slater-Koster hopping integral between the orbitals, ${ }^{22}$ and $\theta_{1 / 2}$ describe the quantization axes (directions) of the spins. The diagonal $2 \times 2$ blocks of Eq. (3) represent the spin-orbit coupling, similar to Eq. (1), whereas the off-

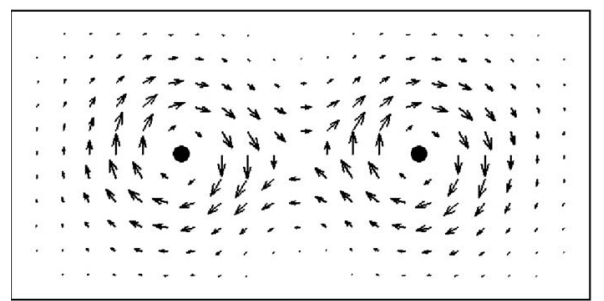

(a)

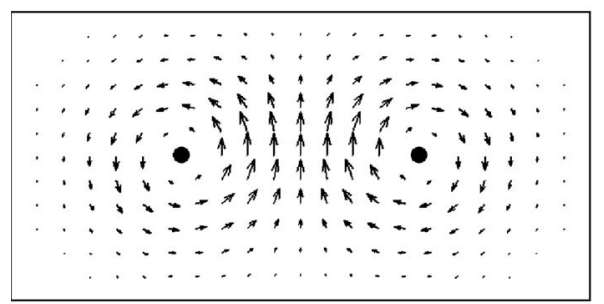

(b)

FIG. 7. Typical tight-binding current densities (arrows) for (a) parallel and (b) antiparallel spin moments. The current density corresponds to electrons with orbital moments that hop from atom to atom.

diagonal $2 \times 2$ blocks describe the usual interatomic hybridization (hopping).

The representation of Eq. (3) corresponds to real wave functions with zero orbital current density $\mathbf{j} \sim i\left(\psi^{*} \nabla \psi\right.$ $\left.-\psi^{*} \nabla \psi\right)$, but the spin-orbit coupling creates a current density $\mathbf{j} \sim \mathbf{r} \times \mathbf{s}$ in each atom, and interatomic hopping then acquires the character of a hopping of current loops. Figure 7 illustrates this hopping in the limit of large orbital moments. In the model of Eq. (3), the current loops are in the plane of the paper, so that $\mathbf{s}$ and $\mathbf{I}$ are perpendicular to the plane. Turning a spin into the plane of the paper destroys the current loops, corresponding to a reduction or "quenching" of the orbital moment. This is the physics behind the $\cos \theta_{1} \cos \theta_{2}$ character of the non-Heisenberg interaction mentioned in Sec. I. Generalized to heteronuclear interactions, the top-left and bottom-right $2 \times 2$ submatrices of Eq. (3) have different spinorbit couplings, and the current-loop hopping leads to a "transfer" of spin-orbit coupling between neighboring atoms. This may also be important for the magnetism of materials such as $\mathrm{HfO}_{2}{ }^{11}$ because the oxygen is important for the moment formation but exhibits virtually no spin-orbit coupling.

In ordinary ferromagnets, the orbital current density due to spin-orbit coupling is usually small and hidden in the derivation of spin Hamiltonians ${ }^{23}$ by formal integration over orbital degrees of freedom. Spin-orbit coupling in semiconductors has a more pronounced effect, as seen, for example, by optical ellipsometry, ${ }^{24}$ and we expect that the hopping of current loops is a widespread phenomenon in dilute magnetic semiconductors. Note that spin-orbit-split bands of nonmagnetic semiconductors are occupied by $\uparrow \downarrow$ electron pairs with opposite orbital moments (opposite current directions), but any spin polarization of external or atomic origin yields a net orbital moment. The currents are macroscopic but originate from atomic spin-orbit interaction, in contrast to the weak "interstitial" spin-orbit coupling of free electrons confined to atomic layers. ${ }^{25}$ 
The mechanism investigated in this paper complements the moment formation and ferromagnetic coupling due to Heisenberg exchange. The latter strongly depends on features such as bond angles and oxygen stoichiometry. For example, oxides tend to effectively involve orbitals that extend beyond third-nearest-neighbor atoms, ${ }^{26}$ and minor changes in the bond angles yield disproportional changes in hopping and exchange. In Eq. (3), Heisenberg exchange is treated rather crudely by postulating rather than calculating the angles $\theta_{1 / 2}$. The detailed investigation of the spinpolarized electronic structure and interatomic exchange as well as their interplay with orbital features remains a challenge for future research. The same is true for the direct observation of spin-orbit coupling and orbital moment: $x$-ray magnetic circular dichroism measurements have been inconclusive so far, which is probably a surface effect.

In conclusion, we have investigated the magnetism of V-doped $\mathrm{SnO}_{2}$ thin films. The films exhibit a significant spontaneous magnetization well above room temperature and a pronounced and temperature-dependent in-plane anisotropy of the magnetic moment. The symmetry axis of the moment anisotropy is incompatible with the rutile crystal structure of $\mathrm{SnO}_{2}$ but consistent with the interstitial site occupancy of the $\mathrm{V}$. The moment anisotropy and its temperature dependence critically depend on spin-orbit coupling. The physics behind the moment anisotropy is atomic current loops formed by the spin-orbit coupling, interacting with the crystalline environment, and hopping onto neighboring atoms.

\section{ACKNOWLEDGMENTS}

The authors are grateful to B. Jones, J. Zhou, L. P. Yue, X. Z. Li, J. Engelen, R. Sabirianov, D. Keavney, M. Schubert, and J. Shi for assistance and helpful discussions. This research is supported by NSF MRSEC, the W. M. Keck Foundation, the Nebraska Research Initiative, and NCMN.
${ }^{1}$ T. Dietl, H. Ohno, F. Matsukura, J. Cibert, and D. Ferrand, Science 287, 1019 (2000).

${ }^{2}$ K. Sato and H. Katayama-Yoshida, Jpn. J. Appl. Phys., Part 2 39, L555 (2000).

${ }^{3}$ K. Ueda, H. Tabata, and T. Kawai, Appl. Phys. Lett. 79, 988 (2001).

${ }^{4}$ P. V. Radovanovic and D. R. Gamelin, Phys. Rev. Lett. 91, 157202 (2003).

${ }^{5}$ T. C. Kaspar, S. M. Heald, C. M. Wang, J. D. Bryan, T. Droubay, V. Shutthanandan, S. Thevuthasan, D. E. McCready, A. J. Kellock, D. R. Gamelin, and S. A. Chambers, Phys. Rev. Lett. 95, 217203 (2005).

${ }^{6}$ J. M. D. Coey, J. Appl. Phys. 97, 10D313 (2005).

${ }^{7}$ M. Venkatesan, C. B. Fitzgerald, J. G. Lunney, and J. M. D. Coey, Phys. Rev. Lett. 93, 177206 (2004).

${ }^{8}$ R. Janisch, P. Gopal, and N. A. Spaddin, J. Phys.: Condens. Matter 17, R657 (2005)

${ }^{9}$ S. B. Ogale, R. J. Choudhary, J. P. Buban, S. E. Lofland, S. R. Shinde, S. N. Kale, V. N. Kulkarni, J. Higgins, C. Lanci, J. R. Simpson, N. D. Browning, S. Das Sarma, H. D. Drew, R. L. Greene, and T. Venkatesan, Phys. Rev. Lett. 91, 077205 (2003).

${ }^{10}$ N. H. Hong and J. Sakai, Physica B 358, 265 (2005).

${ }^{11}$ J. M. D. Coey, M. Venkatesan, P. Stamenov, C. B. Fitzgerald, and L. S. Dorneles, Phys. Rev. B 72, 024450 (2005).

${ }^{12}$ R. Skomski and J. M. D. Coey, Permanent Magnetism (Institute of Physics, Bristol, 1999).

${ }^{13}$ G. Zaránd and B. Jankó, Phys. Rev. Lett. 89, 047201 (2002).

${ }^{14}$ D. S. Chuang, C. A. Ballentine, and R. C. O'Handley, Phys. Rev. B 49, 15084 (1994).

${ }^{15}$ M. Mathews, F. M. Postma, J. C. Lodder, R. Jansen, G. Rijnders, and D. H. A. Blanka, Appl. Phys. Lett. 87, 242507 (2005).

${ }^{16}$ J. E. Dominguez, X. Q. Pan, L. Fu, P. A. Van Rompay, Z. Zhang, J. A. Nees, and P. P. Pronko, J. Appl. Phys. 91, 1060 (2002).

${ }^{17}$ B. K. Rao and P. Jena, Phys. Rev. Lett. 89, 185504 (2002).

${ }^{18}$ F. Kubec and Z. Sroubek, J. Chem. Phys. 57, 1660 (1972).

${ }^{19}$ A. E. Taverner, C. Rayden, S. Warren, A. Gulino, P. A. Cox, and R. G. Egdell, Phys. Rev. B 51, 6833 (1995).

${ }^{20}$ J. S. Smart, Effective Field Theories of Magnetism (Saunders, Philadelphia, 1966).

${ }^{21}$ W. Jones and N. H. March, Theoretical Solid State Physics (Wiley London, 1973), Vol. I.

${ }^{22}$ J. C. Slater and G. F. Koster, Phys. Rev. 94, 1498 (1954).

${ }^{23}$ K. Yosida, Theory of Magnetism (Springer, Berlin, 1996).

${ }^{24}$ M. Schubert, Infrared Ellipsometry on Semiconductor Layer Structures: Phonons, Plasmons and Polaritons (Springer, Berlin, 2004).

${ }^{25}$ R. Skomski, IEEE Trans. Magn. 32, 4794 (1996).

${ }^{26}$ O. K. Andersen and T. Saha-Dasgupta, Phys. Rev. B 62, R16219 (2000). 